\title{
Adrenaline-mediated activation of antibiotic production in Streptomyces highlights catechol as elicitor of specialized metabolism
}

\section{Gilles van Wezel ( $\nabla$ g.wezel@biology.leidenuniv.nl ) \\ Leiden University https://orcid.org/0000-0003-0341-1561}

\section{Doris van Bergeijk}

Institute of Biology, Leiden University

\section{Somayah Elsayed}

Institute of Biology, Leiden University

Chao Du

Microbial Biotechnology, Institute of Biology, Leiden University https://orcid.org/0000-0003-3447-5293

\section{Isabel Nunez Santiago}

Institute of Biology, Leiden University

\section{Anna Roseboom}

Institute of Biology, Leiden University

\section{Le Zhang}

Institute of Biology, Leiden University

\section{Victor Carrion}

Microbial Biotechnology, Institute of Biology, Leiden University

Herman Spaink

Leiden University https://orcid.org/0000-0003-4128-9501

\section{Article}

Keywords: streptomyces, catechol, antibacteria, BGCs, MBT84

Posted Date: August 11th, 2021

DOl: https://doi.org/10.21203/rs.3.rs-715051/v1

License: (c) (1) This work is licensed under a Creative Commons Attribution 4.0 International License. Read Full License

Version of Record: A version of this preprint was published at Communications Chemistry on February 3rd, 2022. See the published version at https://doi.org/10.1038/s42004-022-00632-4. 

1 Adrenaline-mediated activation of antibiotic production in Streptomyces highlights

2 catechol as elicitor of specialized metabolism

3

4 Doris A. van Bergeijk, Somayah S. Elsayed, Chao Du, Isabel Nuñez Santiago, Anna M.

5 Roseboom, Le Zhang, Victor J. Carrión, Herman P. Spaink and Gilles P. van Wezel ${ }^{\star}$

6

7 Institute of Biology, Leiden University, Sylviusweg 72, 2333 BE, Leiden, The Netherlands.

8

$9 \quad$ * author for correspondence. g.wezel@biology.leidenuniv.nl. Tel: +31 715274310.

10

11

12

13

14 


\section{ABSTRACT}

Actinobacteria are a rich source of bioactive molecules, and genome sequencing has shown that the vast majority of their biosynthetic potential has yet to be explored. However, many of their biosynthetic gene clusters (BGCs) are poorly expressed in the laboratory, which prevents discovery of their cognate natural products. To exploit their full biosynthetic potential, better understanding of the signals that promote the expression of BGCs is needed. Here, we show that the human stress hormone epinephrine (adrenaline) elicits antibiotic production by Actinobacteria. Catechol was established as the likely eliciting moiety, since similar responses were seen for catechol and for the catechol-containing molecules dopamine and catechin but not for related molecules. Exploration of the catechol-responsive strain Streptomyces sp. MBT84 using mass spectral networking revealed elicitation of a BGC that produces the angucycline glycosides aquayamycin, urdamycinone B and galtamycin C. Heterologous expression of the catechol-cleaving enzymes catechol 1,2-dioxygenase or catechol 2,3 dioxygenase counteracted the eliciting effect of catechol. Thus, for the first time we show the activation of natural product biosynthesis by a human hormone, leading to the identification of the ubiquitous catechol moiety as elicitor of BGCs for siderophores and antibiotics. 


\section{INTRODUCTION}

The phylum Actinobacteria represents a highly diverse group of bacteria with extraordinary metabolic potential ${ }^{1}$. Their specialized metabolites include most of the clinically used antibiotics along with numerous cancer chemotherapeutics, immunosuppressants, and pesticides, and are therefore of great importance for application as clinical drugs or in agriculture ${ }^{2}$. This metabolic versatility makes Actinobacteria attractive sources for drug discovery, for which there is an urgent need due to the global rise of drug resistance ${ }^{3,4}$. Indeed, molecules of actinobacterial origin continue to be discovered that have important new structural and/or functional features, for example the antifungal cyphomycin ${ }^{5}$, the glycopeptide antibiotic corbomycin $^{6}$, and the angucycline-derived polyketide lugdunomycin ${ }^{7}$.

Genome sequencing revealed that the potential of Actinobacteria as producers of bioactive compounds had been grossly underestimated: their genomes harbor a wealth of biosynthetic gene clusters (BGCs) that specify yet undiscovered natural products ${ }^{8,9}$. However, many of these potentially interesting BGCs are poorly expressed or silent under laboratory growth conditions ${ }^{10}$. To exploit this huge resource of chemical diversity, we need to first understand the triggers and cues that promote their expression. This knowledge can then be translated to eliciting approaches to activate the expression of BGCs and produce their cognate bioactive compounds at high throughput ${ }^{11}$.

One approach to identify the cues that activate BGC expression lies in the understanding of the ecology of host-microbe interactions ${ }^{11}$. Actinobacteria inhabit a wide range of terrestrial and aquatic ecosystems ${ }^{12}$. Within these environments, specialized metabolites play an important role in survival through mediation of resource competition ${ }^{13,14}$, protection against oxidative stresses ${ }^{15}$ and uptake of essential nutrients ${ }^{16}$. This requires careful timing of production and it is therefore likely that environmental signals indicative of specific stresses (such as nutrient levels and the presence of competitors) have been incorporated in the regulation of $\mathrm{BGC}$ expression ${ }^{10,17,18}$. Indeed, nutrient availability and co-culture of Actinobacteria with other microorganisms significantly influence their specialized metabolite production $^{19,20}$. 

wide variety of other organisms, including higher eukaryotes such as plants, insects, marine organisms, and mammals ${ }^{5,11}$. As part of the microbiomes of these hosts, Actinobacteria are exposed to host-associated signaling molecules, many of which will likely influence their specialized metabolism ${ }^{11}$. Indeed, plant stress hormones, such as salicylic acid and jasmonic acid, have been shown to increase the antibiotic activity of endophytic streptomycetes ${ }^{21}$. Since these hormones are excreted by plants under pathogenic stress, it has been hypothesized that their release might represent a "cry for help" through which the plant may activate the antibiotic production of Actinobacteria, in order to counteract a pathogenic attack ${ }^{21}$. Besides plant hormones, also animal stress hormones have been shown to influence bacteria. Specifically, catecholamines, which include the well-known 'fight or flight' hormone adrenaline (also known as epinephrine), influence bacterial growth ${ }^{22,}{ }^{23}$, biofilm formation ${ }^{24}$, and horizontal gene transfer ${ }^{25}$. We hypothesize that animal stress hormones may play a role in the control of antibiotic production of Actinobacteria.

In this work, we demonstrate that the animal stress hormone epinephrine can influence specialized metabolism of streptomycetes, specifically the siderophore production. The catechol moiety showed to be key to this response and this finding could be translated to other host-specific compounds (human and plant) containing a catechol moiety. Catechol by itself enhanced the production of angucyclines. Taken together, these results illustrate that catechol, by itself and as part of host-related signaling molecules, can serve as elicitor of antibiotic production. 


\section{RESULTS}

\section{Epinephrine alters antibiotic production of Actinobacteria}

Actinobacteria live in close association with higher eukaryotes, such as plants and animals. Host stress molecules might play a role in the regulation of actinobacterial specialized metabolism, perhaps reflecting a "cry for help" from host to bacterium ${ }^{21}$. We therefore investigated if animal stress hormones can affect growth and metabolism of Actinobacteria. For this, we analyzed the effect of epinephrine, also known as adrenaline, on a selection of our in-house actinobacterial strain collection that was previously shown to require particular growth conditions for the production of antibiotics ${ }^{26}$. We used the Gram-positive Bacillus subtilis 168 and the Gram-negative Escherichia coli ASD19 as indicator strains. Minimal Medium agar (MM) and Nutrient Agar (NA) plates with or without $100 \mu \mathrm{M}$ epinephrine (bitartrate salt) or tartaric acid (as control for the added bitartrate) were inoculated with spots from spore stocks of different actinobacterial strains. The chosen concentration of $100 \mu \mathrm{M}$ was based on the responsiveness of Actinobacteria to different concentrations of epinephrine (not shown). To see if the addition of epinephrine to the growth media affected the susceptibility of the indicator strains against antibiotics, we added a diffusion disc with ampicillin $(6 \mu \mathrm{g})$ to each plate and tested whether the presence of epinephrine in the growth medium affected the size of the inhibition zone of ampicillin against $E$. coli and B. subtilis. No differences were observed, confirming that addition of epinephrine to the growth medium did not affect the susceptibility of the indicator strains.

On MM, both promotion and inhibition of bioactivity were observed (Fig. S1A). Of these responses, a decrease in bioactivity was observed most frequently (often against both $E$. coli and $B$. subtilis). For some strains, this decrease in bioactivity could be linked to inhibition of growth in presence of epinephrine. On NA, no significant changes in bioactivity where observed except for the elicitation of a large semi-transparent halo surrounding Streptomyces sp. MBT42 by epinephrine (Fig. S1B). 


\section{The catechol moiety is key to the eliciting effect of epinephrine on Streptomyces sp.}

\section{MBT42}

To analyze the eliciting effect of epinephrine in more detail, we selected Streptomyces sp. MBT42, as this strain reproducibly showed a strong response to the hormone when the strain was grown on NA agar plates (Fig. 1A). In particular, we were interested to see whether epinephrine itself or any of its chemical constituents would be responsible for the eliciting effect. To do so, the effects of different catecholamines and structurally related compounds were assessed (Fig. 1B). Besides epinephrine, we also included catechin, dopamine, levodopa and norepinephrine. Interestingly, all of these compounds had an effect similar to or stronger than epinephrine. Since all of the tested compounds contain a catechol moiety, we wondered if catechol (1,2-dihydroxybenzene) may be the moiety primarily responsible for the observed eliciting effect. We therefore tested the effect of catechol itself, and as controls we used several structural analogues, namely the meta isomer resorcinol (1,3-dihydroxybenzene) and the para isomer hydroquinone (1,4-dihydroxybenzene), as well as molecules containing a monohydroxy-substituted benzene ring, such as phenol, phenylephrine and tyramine. Importantly, of these compounds, catechol itself strongly enhanced antibiotic production by Streptomyces sp. MBT42, while the other compounds failed to elicit antibiotic production, although hydroquinone had a mild eliciting effect, close to that of norepinephrine (Fig. 1C). These data strongly suggest that the catechol moiety is key to the response of Streptomyces sp. MBT42 to the stress hormone epinephrine.

\section{Catechol enhances siderophore production in Streptomyces sp. MBT42}

The catechol moiety is well known for its iron-chelating properties. For example, catechin is a strong siderophore ${ }^{27}$. We therefore hypothesized that the addition of catechol-containing molecules might result in lower iron availability, which may trigger siderophore production. To test this hypothesis, we spotted MBT42 spores onto NA with or without $100 \mu \mathrm{M}$ catechol, and overlaid the plates with CAS agar solution after 4 days of growth. An orange halo was formed that is indicative of siderophore production, which was strongly increased in the presence of 
catechol (Fig. 2). These siderophore-related halos matched the semi-transparent halos that were observed when Streptomyces sp. MBT42 was overlaid with B. subtilis.

Siderophore production is suppressed by iron availability. We therefore tested whether the addition of iron could compensate for the effect of catechol on Streptomyces sp. MBT42 Indeed, when $5 \mu \mathrm{M} \mathrm{FeCl}_{3}$ was added to the medium, siderophore production was almost completely inhibited, and this coincided with disappearance of the semi-transparent halo (Fig. 2). The same result was obtained when $\mathrm{FeSO}_{4}$ was added (data not shown). Other metal ions did not have this effect (Fig. 2). Taken together, this shows that catechol acts as an elicitor of siderophores, which is most likely explained by the sequestering of iron.

\section{Catechol elicits the production of bioactive specialized metabolites}

The specificity of the above-described response to the catechol moiety suggested that catechol may be applied as a general elicitor of natural product biosynthesis in Actinobacteria. We therefore tested the effect of catechol on some of the well-characterized Actinobacteria in our collection. Again, we observed both promotion and inhibition of bioactivity in the presence of catechol (Fig. S2). Streptomyces sp. MBT84 responded particularly well to catechol, reproducibly producing strong antibacterial activity against B. subtilis (Fig. 3A) and Staphylococcus aureus (data not shown) in response to catechol. The bioactivity coincided with enhanced production of a yellow/brown pigment (Fig. 3A). However, in this case none of the structurally-related compounds, including epinephrine, increased antibiotic production (Fig. 3B). Also iron did not affect the changes in bioactivity and pigment production, thus we ruled out that siderophore production was the cause of the observed growth inhibition.

To identify the nature of the metabolites produced by Streptomyces sp. MBT84 in response to catechol, the strain was streaked on MM agar plates and the metabolites were then extracted from the spent agar after five days of growth using ethyl acetate (EtOAc). The crude extracts of the catechol-grown cultures showed increased bioactivity as compared to those grown under control conditions, while media blanks showed no activity (data not shown). Liquid chromatography-mass spectrometry (LC-MS) analysis revealed multiple peaks in the 
chromatogram that were substantially overrepresented in the samples obtained from the catechol-grown cultures (Fig. 3C). The metabolomic profile of Streptomyces sp. MBT84 grown with and without catechol was compared through a volcano plot generated using MetaboAnalyst $^{28}$ (Fig. 3D). In the volcano plot, the majority of the mass features were significantly upregulated in catechol-grown cultures. The most notable ones were those with $m / z$ values 469.1494 and 579.1864 , which represented several highly intense mass features sharing the same exact masses but with different retention times. Additionally, multiple insource fragments were among the mass features which were significantly upregulated with catechol. The mass features related to $\mathrm{m} / \mathrm{z} 469.1494$ and 579.1864 are likely due to yellowishcolored metabolites, since they showed in the ultraviolet (UV) chromatogram corresponding peaks having UV absorption maxima of $420-440 \mathrm{~nm}$. This corresponds well to the observed increase in yellow pigmentation when Streptomyces sp. MBT84 was grown in the presence of catechol.

To identify which $m / z$ values correlated to the increased bioactivity of Streptomyces sp. MBT84, crude extracts were analyzed using at-line nanofractionation coupled to LC-MS, using a method described previously ${ }^{29}$. In brief, the LC effluent is split post-column, after which one tenth of the flow goes to the MS, while the rest was fractionated into a 384-well plate (6 seconds/well). Following LC solvent evaporation, the antibacterial activity of the collected nanofractions is assessed in a resazurin reduction assay and directly correlated to the mass spectral peaks. Reconstruction of the bioactivity chromatograms revealed multiple negative maxima, reflecting bioactivity. After alignment to the LC-MS chromatogram, the $\mathrm{m} / \mathrm{z}$ values of the bioactive peaks were determined, which included those which correspond to the yellowish metabolites ( $\mathrm{m} / \mathrm{z} 469.149$ and 579.186) (Fig. 4). In the extracted ion chromatogram of $\mathrm{m} / \mathrm{z}$ 469.15, the multiple peaks observed were either due to quasi-molecular ions (like peaks number 7 and 9), or due to fragment ions of $\mathrm{m} / \mathrm{z} 487.16$ (like peaks number 1 and 2 ). The same pattern was observed for the peaks due to $m / z 579.19$, which were mostly fragment ions of $m / z 597.20$. 
To gain more insight into which metabolites were induced by catechol and whether these were structurally related, molecular networking was employed using the Global Natural Products Social molecular networking (GNPS) web tool. This generates a network wherein molecules with related scaffolds cluster together ${ }^{30}$. Due to the presence of multiple isomeric metabolites, we used the classical molecular networking workflow while enabling the MSCluster algorithm ${ }^{30,31}$. This allows to merge all mass features having similar MS and MS/MS spectra into one node, regardless of their different retention times. A network representing the ions detected in the crude extract of Streptomyces sp. MBT84 grown with and without catechol was constructed, revealing 258 nodes clustered in 21 spectral families (Fig. 5). Within the biggest spectral family, dereplication based on matching MS/MS spectra against the GNPS spectral library annotated several $\mathrm{m} / \mathrm{z}$ values as being known metabolites belonging to the class of angucyclines, namely aquayamycin (1), dehydroxyaquayamycin (2), and urdamycinone B (3). Additionally, two structurally related compounds $(\mathbf{4 , 5})$ as well as an angucycline-like compound (6), were annotated. This angucyclines spectral family was more abundant in the presence of catechol and included the majority of the mass features that were correlated to bioactivity by nanofractionation (Fig. 5).

To ascertain that this network indeed consisted of angucycline glycoside-like compounds, we isolated the molecules with $\mathrm{m} / \mathrm{z} 561.1760$ (7) and 579.1866 (8). For this, Streptomyces sp. MBT84 was grown confluently on $12 \times 12 \mathrm{~mm} \mathrm{MM}$ agar plates with $50 \mu \mathrm{M}$ catechol and the metabolites were extracted with EtOAc. Following chromatographic isolation, 7 (red amorphous powder) and 8 (yellow amorphous powder) were obtained. The final structures of $\mathbf{7}$ and $\mathbf{8}$ were elucidated using nuclear magnetic resonance spectroscopy (NMR) (Supplemental data). Based on that, $\mathbf{7}$ was identified as galtamycin $\mathrm{C}$, which has previously been isolated from an intertidal sediments-derived Streptomyces $\mathrm{sp}^{32}$. As for $\mathbf{8}$, it was found to be 1-oxo-3-hydroxy-3,4-dihydro-2H-galtamycin $\mathrm{C}$ based on the different $2 \mathrm{D} \mathrm{NMR}$ correlations observed in comparison with 7 , together with the molecular formula and degrees of unsaturation obtained from its accurate mass (Supplemental data). To our knowledge 8 has not been previously described, and was thus designated as galtamycin D. Taken together, our 
data show that catechol enhances the biosynthesis of angucycline glycosides in Streptomyces sp. MBT84.

\section{Identification of the angucycline glycoside BGC in Streptomyces sp. MBT84}

To identify the gene cluster responsible for the biosynthesis of the angucycline glycosides in Streptomyces sp. MBT84, we obtained its full genome sequence using PacBio sequencing (GenBank accession number: JAHTGP000000000). A draft genome was assembled resulting in 3 contigs (Table S3). Analysis using antiSMASH $6.0^{33}$ revealed 21 putative BGCs (Table S4) of which BGC1.4 shares $>87 \%$ identity with the saquayamycin A BGC, and was therefore most likely responsible for the production of the angucycline glycosides (Fig. 6A).

We have previously shown the applicability of quantitative proteomics combined with metabolomics to connect BGCs to the natural product(s) they specify ${ }^{34}$. Therefore, MS-based quantitative proteomic analysis was performed on total protein samples obtained from Streptomyces sp. MBT84 grown on MM agar plates with or without $100 \mu \mathrm{M}$ catechol covered with a cellophane disc. After five days of growth, the biomass was harvested and snap-frozen significantly differentially expressed between catechol-grown and control cultures (FDRadjusted $p$-value $<0.1$; Fig. $6 \mathrm{~B})$. Proteins belonging to five of the 21 putative BGCs annotated by the antiSMASH algorithm were expressed under the chosen conditions. However, only the biosynthetic proteins of BGC1.4 were mostly significantly upregulated in catechol-grown cultures, which provided validation of the involvement of this BGC in the observed change in bioactivity induced by catechol (Fig. 6B and C). Surprisingly, the minimal polyketide synthase (PKS) enzymes responsible for the generation of the initial angucycline or angucyclinone backbone ${ }^{35}$, namely two ketosynthase units $(\mathrm{KS} \alpha$ and $\mathrm{KS} \beta)$ and an acyl carrier protein, were not significantly differentially expressed (Fig. 6C).

To confirm that BGC1.4 was indeed responsible for the production of the angucycline glycosides, we knocked down transcription of the gene for the KS $\beta$ of the minimal PKS using 
CRISPR-dCas9 interference ${ }^{36,37}$. The knock-down was enforced from the CRISPRi construct pGWS1517 that expresses a spacer targeting the non-template strand of the gene for KS $\beta$.

Pigment production was strongly inhibited in the strain with reduced expression of KS $\beta$, while no inhibition was seen in the control strain harboring a construct (pGWS1516) that targets the template strand (Fig. S3). Metabolomics analysis revealed that the production of the angucycline glycosides was significantly reduced when the expression of KS $\beta$ was inhibited (Fig. S3). All the evidence together proves that indeed BGC1.4 specifies the angucyclines.

\section{Expression of catechol-degrading enzymes counteracts antibiotic elicitation}

257

258

259

260

261

262

Various microorganisms (e.g. Pseudomonades) enzymatically degrade aromatic compounds $^{38}$. To establish whether catechol itself is essential for the eliciting effect, we expressed two enzymes in Streptomyces sp. MBT84 that degrade catechol. Catechol can be cleaved into cis, cis-muconic acid by catechol 1,2-dioxygenase (C12O) (ortho-pathway) or into 2-hydroxymuconic semialdehyde by catechol 2,3-dioxygenase (C23O) (meta-pathway) (Fig. S4) ${ }^{38}$. We wondered whether these degradation products would have a similar eliciting effect on angucycline production or whether the effect of catechol would disappear once degraded. To this end, we separately cloned the catA1 gene encoding $\mathrm{C} 12 \mathrm{O}$ from Pseudomonas putida KT2440 and the $x y / E$ gene encoding C23O from the promoter-probe vector plJ4083 39 onto the multi-copy vector pWHM3. The strong constitutive promoter SF14 ${ }^{40}$ was used to drive expression. The resulting constructs pGWS1519 and pGWS1520 were introduced in Streptomyces sp. MBT84 via protoplast transformation, whereby the empty vector was used as a control. Of each recombinant strain (named MBT84-pWHM3, MBT84-C12O, MBT84C230) three independent transformants were tested for their ability to respond to catechol.

The transformants were grown for five days on MM agar plates with and without 100 $\mu \mathrm{M}$ catechol, and the metabolites were extracted as described above. While catechol induced a clear increase in yellow pigmentation in MBT84 harboring the control plasmid, such induction was no longer visible when MBT84-C12O was grown in the presence of catechol (Fig. S5A). In strain MBT84-C23O, the effect of catechol on the yellow pigmentation was strongly reduced, 
but in some transformants still some induction was observed. The visual assessment of

277 antibiotic production was confirmed by detailed metabolomic analysis (Fig. S5B). Indeed, while catechol again elicited the production of metabolites in the control strain, catechol did not alter the metabolic profile of transformants expressing the catechol-degrading enzyme $\mathrm{C} 12 \mathrm{O}$ or C230. However, for the latter, the volcano plot showed a similar skewing to the upregulated side as the control, which suggests a reduced response.

To analyse the expression of the catechol dioxygenases and of BGC1.4 in the recombinant strains, we used quantitative proteomic analysis as described previously ${ }^{41}$. C12O and C23O were expressed individually in the respective recombinant strains (Fig. S5C). To study the expression profile of BGC1.4 of each transformant (or WT replicate), a heatmap of the $\log _{2}$ fold changes in expression between catechol and control was generated. Additionally, violin plots were generated to show the distribution of the expression differences of BGC1.4 proteins (Fig. S5D). The small sample size and variability between the different transformants, did not allow for a thorough statistical analysis. However, while catechol induced a similar upregulation of the expression of BGC1.4 in MBT84-pWHM3 compared to WT, this pattern was no longer visible in MBT84-C12O and MBT84-C23O. These results catechol is the elicitor and not one of its degradation products.

\section{DISCUSSION}

Actinobacteria harbor many BGCs that are not or poorly expressed under laboratory growth conditions. A better understanding of the environmental signals that influence BGC expression as well as the regulatory pathways involved, will allow us to further explore actinobacterial specialized metabolism for therapeutic uses. Our work reveals catechol as elicitor of siderophore and antibiotic production in different streptomycetes. Specifically, catechol enhanced the expression of an angucycline BGC in Streptomyces sp. MBT84. Angucyclines constitute the largest group of aromatic polyketides and are well known for their structural diversity and their therapeutic potential as anticancer and antibiotic compounds ${ }^{35}$. Our recent discovery of the lugdunomycins and a range of novel angucyclines revealed that this 
extensively explored family of polyketides still has potential for drug discovery ${ }^{7}$. Indeed, the new compound galtamycin D (8) described in this study is another example that many angucyclines and derivatives are still to be discovered. Environmental cues may not only elicit the expression of (cryptic) biosynthetic pathways, but also of previously unseen branches of known pathways. Indeed, co-cultivation of the model streptomycete Streptomyces coelicolor A3(2) with Aspergillus niger elicited the production of GTRI-02, which is produced by the actinorhodin biosynthetic pathway ${ }^{42}$. The type II polyketide actinorhodin has been studied extensively by many laboratories for over 60 years, and the surprising discovery of an entirely new branch of its biosynthetic pathway underlines the importance of microbial interactions for drug discovery. Similarly, supplementation of catechol to growth conditions might offer a new strategy to further explore the immense chemical space of angucyclines and other polyketides. Preliminary experiments in our laboratory revealed many elicited mass features in the metabolome of other Streptomyces species grown in presence of catechol (not shown). Our data indicate that these mass features belong to non-ribosomal peptides and aminoglycoside antibiotics, which we are currently investigating. Thus, supplementation of catechol to growth media may allow for prioritization of bioactive metabolites for isolation from metabolite extracts which are often very complex mixtures.

To coordinate appropriate production of specialized metabolites, Actinobacteria have evolved a vast array of complex, multi-level regulatory pathways of which many remain to be elucidated. The identification of a signal that influences specialized metabolism can provide a first step towards the elucidation of a pathway, as was illustrated by the discovery that GlcNAc had a stimulating effect on antibiotic production of many Streptomyces sp. ${ }^{20}$. Elucidating the signal transduction pathway identified DasR as pleiotropic transcriptional repressor of several pathway-specific activators, including Actll-ORF4 and RedD, whereby GlcN-6P acts as ligand for Das ${ }^{20}$. We propose that the presence of catechol-containing molecules results in lower iron availability, which triggers siderophore production. However, the mechanism via which catechol impacts angucycline production in Streptomyces, remains yet unexplained. Our experiments show that degradation of catechol will annihilate its effect. Heterologous 
expression of the catechol-cleaving enzymes $\mathrm{C} 12 \mathrm{O}$ or C23O in Streptomyces sp. MBT84 strongly reduced its response to catechol. This strongly suggests that catechol is the elicitor and not one of its degradation products. Of the two enzymes, C23O had slightly less impact than $\mathrm{C} 12 \mathrm{O}$, whereby the latter fully counteracted the effect of catechol. Our proteomics experiments show that both enzymes are expressed well, and we anticipate that the difference may lie in the nature of their degradation products. $\mathrm{C} 23 \mathrm{O}$ is responsible for the extradiol ring cleavage of catechol, producing 2-hydroxymuconic semialdehyde in which the 1,2 di-hydroxy architecture of catechol is preserved, while $\mathrm{C} 12 \mathrm{O}$ does not maintain this scaffold. This could indicate that specific protein interactions are involved in the response to catechol and might also explain why Streptomyces sp. MBT84 only responds to catechol. Catecholamines are protonated at neutral $\mathrm{pH}$, which could interfere with protein binding. The next step will be to elucidate the signal transduction pathway for catechol, and identify the key regulatory network it targets inside the cell. various catechol-containing host-derived signaling molecules: the mammalian stress hormones epinephrine, dopamine and norepinephrine (collectively known as catecholamines), their precursor levodopa, and the plant metabolite catechin. Interestingly, high throughput elicitor screening (HiTES) technology identified the plant-derived piceatannol as elicitor of the

Catechol broadly impacts specialized metabolism, as exemplified by its effect on the biosynthesis of angucyclines and siderophores. Production of the latter was enhanced by expression of a BGC for a cryptic NRPS ${ }^{43}$. Considering the data presented in our work, we hypothesize that this response might also be mediated through the catechol moiety that is present in piceatannol.

Siderophores are important mediators of interactions between members of microbial communities and the eukaryotic hosts they inhabit ${ }^{16,}{ }^{44}$. We show that host-associated molecules with a catechol moiety increase siderophore production, resulting in decreased growth of $B$. subtilis. Potentially, catechol-mediated elicitation of siderophore production may influence the composition of microbial communities in the environment or within a host. This may have beneficial effects and, for example, provide protection against pathogens, or 
opposite, cause a disbalance and allow opportunistic pathogens to invade. However, this study has only focused on the effects of such molecules in vitro and on individual bacteria. Whether these responses occur in situ and how they affect microbial communities remains to be tested.

\section{CONCLUSION}

Collectively, our results show that catechol, by itself and as molecular signature of signaling molecules, can alter antibiotic production in Streptomyces, likely via multiple modes of action. Understanding the mechanisms involved is of great interest as they may offer tools to access silent and/or cryptic specialized metabolites from Actinobacteria. Additionally, they may provide insights in the interactions that may take place within microbial communities and between host and bacterium.

\section{METHODS}

\section{Bacterial strains, growth conditions and antimicrobial activity assay}

All media and routine Streptomyces techniques have been described previously ${ }^{45}$. The bacterial strain collection used in this study were obtained from the Leiden MBT strain collection. Streptomyces sp. MBT42 and Streptomyces sp. MBT84 have been isolated from soil samples collected from Cheverny (France) and the QinLing Mountains (Shanxi province, China) ${ }^{26}$ respectively. Bacillus subtilis 168 and Escherichia coli ASD1946 were used as indicator strains for antimicrobial activity and were cultured in LB media at $37^{\circ} \mathrm{C}$.

Antimicrobial activity assays were conducted using the double-layer agar method. Strains were spotted on minimal medium agar plates (MM) supplemented with $0.5 \%$ mannitol and $1 \%$ glycerol (w/v) as non-repressing carbon sources, and nutrient agar (NA) (Difco) plates, using a pin replicator. For individual testing of strains, $2 \mu \mathrm{L}$ spore stock was manually spotted. Growth media were supplemented with $25 \mathrm{mM}$ TES buffer and $100 \mu \mathrm{M}$ of either (+)-catechin hydrate (Sigma-Aldrich, CAS\# 225937-10-0) , catechol (Sigma-Aldrich, CAS\# 120-80-9), dopamine hydrochloride (Sigma-Aldrich, CAS\# 62-31-7), (-)-epinephrine (+)-bitartrate salt (Sigma-Aldrich, CAS\# 51-42-3), hydroquinone (Sigma-Aldrich, CAS\# 123-31-9), levodopa 
(Sigma-Aldrich, CAS\# 59-92-7), norepinephrine bitartrate monohydrate (MCE, CAS\# 10834118-0), phenol (VWR, CAS\# 108-95-2), (R)-(-)-phenylephrine hydrochloride (Sigma-Aldrich, CAS\# 61-76-7), tartaric acid (Sigma-Aldrich, CAS\# 87-69-4), or tyramine hydrochloride (Sigma-Aldrich, CAS\# 60-19-5).

After four days of incubation at $30^{\circ} \mathrm{C}$, plates were overlaid with soft LB agar $(1.8 \% \mathrm{w} / \mathrm{v}$ agar) containing one of the indicator strains $(2 \% \mathrm{v} / \mathrm{v})$ pre-grown in liquid LB to exponential phase $\left(\mathrm{OD}_{600}=0.4-0.6\right)$ and incubated overnight at $37^{\circ} \mathrm{C}( \pm 18$ hours). The following day, antibacterial activity was determined as the ratio between the inhibition zone diameter and the spot diameter.

\section{Chrome azurol S (CAS) assay for siderophore detection}

The medium for $1 \mathrm{~L}$ CAS was prepared according to the method of Schwyn and Neilands $(1987)^{47}$ without the addition of nutrients: $60.5 \mathrm{mg}$ CAS, $72.9 \mathrm{mg}$ hexadecyltrimethyl ammonium bromide (HDTMA-Br), $30.24 \mathrm{~g}$ piperazine-1,4-bis(2-ethanesulfonic acid) (PIPES) and $10 \mathrm{~mL}$ of $1 \mathrm{mM} \mathrm{FeCl}{ }_{3} \cdot 6 \mathrm{H}_{2} \mathrm{O}$ in $10 \mathrm{mM} \mathrm{HCl}$. Agarose $(0.9 \% \mathrm{w} / \mathrm{v})$ was used as gelling agent. The CAS agar solution was overlaid onto the spots of Streptomyces sp. MBT42. Following incubation overnight at $30^{\circ} \mathrm{C}$ ( \pm 18 hours) the plates were examined visually for halos.

\section{Bioactivity of crude extracts and metabolite profiling}

Streptomyces sp. MBT84 was grown confluently on MM agar plates with and without $100 \mu \mathrm{M}$ catechol. After five days of growth, the agar plates were cut into small pieces, soaked overnight in ethyl acetate (EtOAc) to extract the metabolites, evaporated at room temperature and dissolved in $\mathrm{MeOH}$ to a final concentration of $20 \mathrm{mg} / \mathrm{mL}$. $10 \mu \mathrm{L}$ was spotted onto a sterile filter disc placed onto a soft agar layer inoculated with $B$. subtilis 168 . As controls, $10 \mu \mathrm{L} \mathrm{MeOH}$ and $6 \mu \mathrm{L}$ of $1 \mathrm{mg} / \mathrm{mL}$ ampicillin solutions were used. Following incubation overnight at $37^{\circ} \mathrm{C}( \pm 18$ hours), the plates were examined visually for halos.

For liquid chromatography-tandem mass spectrometry (LC-MS/MS) analysis, the dry extracts were dissolved in $\mathrm{MeOH}$ to a final concentration of $1 \mathrm{mg} / \mathrm{mL}$. LC-MS/MS acquisition was 
performed using Shimadzu Nexera X2 ultra high performance liquid chromatography (UPLC) system, with attached photodiode array detector (PDA), coupled to Shimadzu 9030 QTOF mass spectrometer, equipped with a standard electrospray ionisation (ESI) source unit, in which a calibrant delivery system (CDS) is installed. A total of $2 \mu \mathrm{L}$ was injected into a Waters Acquity HSS $\mathrm{C}_{18}$ column $(1.8 \mu \mathrm{m}, 100 \AA, 2.1 \times 100 \mathrm{~mm})$. The column was maintained at 30 ${ }^{\circ} \mathrm{C}$, and run at a flow rate of $0.5 \mathrm{~mL} / \mathrm{min}$, using $0.1 \%$ formic acid in $\mathrm{H}_{2} \mathrm{O}$, and $0.1 \%$ formic acid in acetonitrile $(A C N)$ as solvents $A$ and $B$, respectively. The gradient used was $5 \% B$ for 1 min, 5-85\% B for $9 \mathrm{~min}, 85-100 \% \mathrm{~B}$ for $1 \mathrm{~min}$, and $100 \% \mathrm{~B}$ for $4 \mathrm{~min}$. The column was reequilibrated to $5 \% \mathrm{~B}$ for $3 \mathrm{~min}$ before the next run was started. The PDA acquisition was performed in the range of $200-600 \mathrm{~nm}$, at $4.2 \mathrm{~Hz}$, with $1.2 \mathrm{~nm}$ slit width. The flow cell was maintained at $40^{\circ} \mathrm{C}$.

All the samples were analyzed in positive polarity, using data dependent acquisition mode. In this regard, full scan MS spectra (m/z 100-1700, scan rate $10 \mathrm{~Hz}$, ID enabled) were followed by two data dependent MS/MS spectra (m/z 100-1700, scan rate $10 \mathrm{~Hz}$, ID disabled) for the two most intense ions per scan. The ions were fragmented using collision induced dissociation (CID) with fixed collision energy (CE $20 \mathrm{eV}$ ), and excluded for $1 \mathrm{~s}$ before being re-selected for fragmentation. The parameters used for the ESI source were: interface voltage $4 \mathrm{kV}$, interface temperature $300{ }^{\circ} \mathrm{C}$, nebulizing gas flow $3 \mathrm{~L} / \mathrm{min}$, and drying gas flow $10 \mathrm{~L} / \mathrm{min}$.

\section{LC-MS based comparative metabolomics}

Raw data obtained from LC-MS analysis were converted to mzXML centroid files using Shimadzu LabSolutions Postrun Analysis. The files were imported into Mzmine 2.53 for data processing ${ }^{48}$. Unless stated otherwise, $\mathrm{m} / \mathrm{z}$ tolerance was set to $0.002 \mathrm{~m} / \mathrm{z}$ or $10.0 \mathrm{ppm}$, RT tolerance was set to $0.05 \mathrm{~min}$, noise level was set to $2.0 \mathrm{E} 2$ and the minimum absolute intensity was set to 5.0E2. Raw data were cropped to RT 0.5-12 min. Mass ion peaks were detected (positive polarity, mass detector: centroid) and their chromatograms were built using ADAP

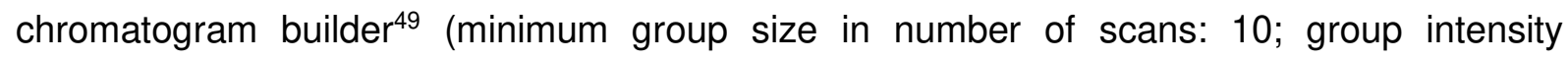
threshold: 2.0E2). The detected peaks were smoothed (filter width: 9), and the chromatograms 
were deconvoluted (algorithm: local minimum search; chromatographic threshold: $85 \%$; search minimum in $\mathrm{RT}$ range: 0.05 ; minimum relative height: $1 \%$; minimum ratio of peak top/edge: 2 ; peak duration: 0.03-2.00 $\mathrm{min}$ ). The detected peaks were deisotoped (monotonic shape; maximum charge: 2; representative isotope: most intense). Peak lists from different extracts were aligned (weight for RT = weight for $\mathrm{m} / \mathrm{z}=20$; compare isotopic pattern with a minimum score of $50 \%$ ). Missing peaks detected in at least one of the samples were filled with the gap filling algorithm (RT tolerance: $0.1 \mathrm{~min}$ ). Among the peaks, we identified fragments (maximum fragment peak height: $50 \%)$, adducts $\left([\mathrm{M}+\mathrm{Na}]^{+},[\mathrm{M}+\mathrm{K}]^{+},\left[\mathrm{M}+\mathrm{NH}_{4}\right]^{+}\right.$, maximum relative adduct peak height: $3000 \%$ ) and complexes (Ionization method: $[\mathrm{M}+\mathrm{H}]^{+}$, maximum complex height: $50 \%)$. Duplicate peaks were filtered. Artifacts caused by detector ringing were removed $(\mathrm{m} / \mathrm{z}$ tolerance: $1.0 \mathrm{~m} / \mathrm{z}$ or $1000.0 \mathrm{ppm})$. The aligned peaks were exported to a MetaboAnalyst file. In Excel, features that were not consistently present with an intensity higher than 8000 in all samples were removed from the file. Additionally, all features that originate from the culture medium were removed by retaining only features with an average peak intensity of at least 50 times greater in the bacterial extracts than in the culture medium extracts. The resulting peak list was uploaded to MetaboAnalyst ${ }^{28}$ for statistical analysis. Log transformation with pareto scaling was applied to the data. Differences with a twofold change and an FDR-adjusted $p$ value $<0.1$ were considered statistically significant (unless otherwise stated). Based on these criteria, volcano plots and heat maps were generated.

\section{MS/MS-based molecular networking and dereplication}

MS/MS raw data (obtained from Shimadzu 9030 QTOF MS) were converted to a 32-bit mzML file using MSConvert (ProteoWizard) and a molecular network was assembled using the online Global Natural Product Social Molecular Networking (GNPS) tool ${ }^{30}$. Both the precursor ion and the MS/MS fragment ion mass tolerance were set to $0.02 \mathrm{Da}$. The minimum cosine score was set to 0.7 and the minimum matched peaks set to 5 . The MSCluster algorithm was run with a minimum cluster size of 2 spectra. The spectra in the network were searched against the GNPS spectral libraries. For this, the precursor ion and the MS/MS fragment ion mass 
tolerance were set to $0.5 \mathrm{Da}$. Matches between network spectra and library spectra required a minimum score of 0.7 and at least 6 matched peaks. Cytoscape 3.5.1 was used for visualization of the generated molecular networks. The edge thickness was set to represent the cosine score, with thicker lines indicating higher similarity between nodes. LC-MS/MS data were deposited in the MassIVE Public GNPS data set (MSV000087784). The molecular networking job in GNPS can be found at

https://gnps.ucsd.edu/ProteoSAFe/status.jsp?task=78cfa392f9c94ac0a110dc682a2d8e6f.

The annotated MS/MS spectra were deposited in the GNPS spectral library for galtamycin C (CCMSLIB00006675753) and the new compound 8 (CCMSLIB00006675754).

\section{At-line nanofractionation and bioactivity assessment}

Liquid chromatography separation, subsequent at-line nanofractionation and parallel mass spectrometry analysis were performed as previously described ${ }^{29}$. Each sample was measured in duplicate. The extracts were dissolved in $\mathrm{MeOH}$ to a final concentration of $4 \mathrm{mg} / \mathrm{mL}$, and 10 $\mu \mathrm{L}$ were injected into Waters Acquity UPLC system with attached PDA, equipped with XBridge Peptide $\mathrm{BEH} \mathrm{C}_{18}$ column $(5 \mu \mathrm{m}, 300 \AA, 4.6 \times 100 \mathrm{~mm})$. The flow rate used was $0.6 \mathrm{~mL} / \mathrm{min}$. Solvent $A$ was $0.1 \%$ formic acid in $\mathrm{H}_{2} \mathrm{O}$ and; solvent $B$ was $0.1 \%$ formic acid in ACN. The gradient used was $0-50 \%$ B for $20 \mathrm{~min}, 50-90 \%$ for $5 \mathrm{~min}, 90 \%$ for $5 \mathrm{~min}, 90-100 \%$ for $1 \mathrm{~min}$ and $100 \%$ for $10 \mathrm{~min}$. After the flow was split in a $1: 9$ ratio, the smaller fraction was fed to a Thermo Instruments MS system (LTQ Orbitrap XL, Bremen, Germany) equipped with an ESI source. The following ESI parameters were used: capillary voltage $5 \mathrm{~V}$, spray voltage $3.5 \mathrm{kV}$, capillary temperature $300{ }^{\circ} \mathrm{C}$, auxiliary gas flow rate 10 arbitrary units, and sheath gas flow rate 50 arbitrary units. MS spectra were acquired in the Orbitrap in positive mode at a mass range of $100-2000 \mathrm{~m} / \mathrm{z}$, and FT resolution of 30,000 . The larger fraction eluted from the UPLC was sent to the chip-based nano-electrospray ionization source/fractionation robot (NanoMate Triversa, Advion BioSciences). The delay was determined as 0.3 min by injection $1 \mu \mathrm{L}$ of 0.5 $\mathrm{mg} / \mathrm{mL}$ ampicillin dissolved in water. Dried plates with the nanofractionated extract were tested for antibacterial activity in a resazurin reduction assay against $B$. subtilis 168 following the 
method described by Mladic et al. (2018) ${ }^{29}$. The bioactivity chromatograms were plotted in GraphPad Prism 9 software.

\section{Large-scale fermentation and isolation of metabolites 7 and 8}

Streptomyces sp. MBT84 was grown on MM agar plates supplemented with $50 \mu \mathrm{M}$ catechol, $1 \%$ glycerol and $0.5 \%$ mannitol at $30{ }^{\circ} \mathrm{C}$ for five days. Agar plates were cut into small pieces and soaked in EtOAc to extract metabolites as described earlier. The solvent was subsequently evaporated under reduced pressure at $40{ }^{\circ} \mathrm{C}$ to obtain $1.6 \mathrm{~g}$ crude extract. This extract was adsorbed onto $1.6 \mathrm{~g}$ silica gel (pore size $60 \AA$, 70-230 mesh, Sigma Aldrich), and loaded on a silica column, followed by gradient elution using mixtures of $n$-hexane, EtOAc, and $\mathrm{MeOH}$. One of the fractions that eluted with 50\% EtOAc : 50\% n-hexane was combined with the fraction that eluted with $75 \%$ EtOAc : $25 \%$ n-hexane, and reconstituted in acetonitrile. This fraction was further purified using a Waters preparative HPLC system comprised of 1525 pump, 2707 autosampler, and 2998 PDA detector. The pooled fraction was injected into a SunFire $\mathrm{C}_{18}$ column $(10 \mu \mathrm{m}, 100 \AA, 19 \times 150 \mathrm{~mm})$. The column was run at a flow rate of $12.0 \mathrm{~mL} / \mathrm{min}$, using solvent $\mathrm{A}\left(\mathrm{dH}_{2} \mathrm{O}\right)$ and solvent $\mathrm{B}$ (acetonitrile), and a gradient of $70-100 \%$ B over 20 min to yield 7 (3.4 mg) and 8 (1.9 mg).

Galtamycin D (8): yellow amorphous powder; UV (LC-MS) $\lambda \max 222,266$, and $441 \mathrm{~nm}$; HRESIMS $m / z 579.1866[\mathrm{M}+\mathrm{H}]^{+}$(calcd for $\left.\mathrm{C}_{31} \mathrm{H}_{31} \mathrm{O}_{11}, 579.1861\right) ;{ }^{1} \mathrm{H}$ and ${ }^{13} \mathrm{C}$ NMR data (Table S5).

\section{NMR measurements}

NMR measurements for the purified compounds were recorded on Bruker Ascend 850 NMR spectrometer (Bruker BioSpin $\mathrm{GmbH}$ ), equipped with a $5 \mathrm{~mm}$ cryoprobe. The samples were measured in DMSO- $d_{6}$ in a $3 \mathrm{~mm}$ NMR tube through the use of an adapter. The spectra were referenced using the solvent residual peak and processed in MestReNova software. 


\section{Genome sequencing, assembly and annotation}

MBT84 was grown in $25 \mathrm{~mL}$ of YEME supplemented with $0.5 \%$ glycine and $5 \mathrm{mM} \mathrm{MgCl}_{2}$ and cultivated at $30{ }^{\circ} \mathrm{C}$ with $200 \mathrm{rpm}$ shaking speed. Genomic DNA was isolated by phenolchloroform extraction as described previously ${ }^{45}$. Genome sequencing was performed using Pacbio Sequel RSII at DNA link Sequencing Lab - South Korea. Raw sequences were demultiplexed with Lima 1.9.0 to produce CCS reads which were converted to fastq with bam2fastq 1.3.0. The assembly was performed with Flye $2.5^{50}$. The genome has been deposited at GenBank under accession number JAHTGP000000000. Biosynthetic gene clusters were identified using the genome mining tool antiSMASH $6.0^{33}$.

\section{Proteomics sample preparation}

MM agar plates with and without $100 \mu \mathrm{M}$ catechol were covered with cellophane. MBT84 spores were spread on the plates using glass beads and incubated for five days at $30{ }^{\circ} \mathrm{C}$. Biomass was scraped off and snap-frozen in liquid nitrogen, lysed in a precooled TissueLyser adaptor (Qiagen, The Netherlands) and proteins extracted using lysis buffer [4\% SDS, $100 \mathrm{mM}$ tris- $\mathrm{HCl}(\mathrm{pH} 7.6), 50 \mathrm{mM}$ EDTA]. The final peptide concentration was adjusted to $40 \mathrm{ng} \cdot \mu \mathrm{L}^{-1}$ using sample solution ( $3 \%$ acetonitrile, $0.5 \%$ formic acid) for analysis.

\section{Proteomics of catechol-grown cultures (proteomining)}

For the analysis of the expression of biosynthetic enzymes in response to catechol, we applied natural product proteomining ${ }^{34}$. Samples were prepared from Streptomyces surface-grown cultures grown with or without catechol. Desalted peptide solutions were injected into Waters nanoAcquity UPLC system equipped with a Waters HSS T3 $\mathrm{C}_{18}(1.8 \mu \mathrm{m}, 100 \AA, 75 \mu \mathrm{m} \times 250$ $\mathrm{mm}$ ). A gradient from $1 \%$ to $40 \%$ acetonitrile in water (with added $0.1 \% \mathrm{FA}$ ) over 110 min was applied. Online MS/MS analysis was done using a Waters Synapt G2-Si HDMS mass spectrometer with a UDMS ${ }^{\mathrm{E}}$ method set up as described previously ${ }^{51}$. [Glu1]-fibrinopeptide B was used as a lock mass compound and sampled every $30 \mathrm{~s}$. Raw data from all samples were first analysed using the vender software ProteinLynx Global SERVER (PLGS, version 3.0.3, 
waters, USA). The resulting dataset was imported in ISOQuant version $1.8^{51}$ for label-free quantification. TOP3 quantification was filtered to remove identifications that had an average value of less than 4000 , in all replicates. This led to the removal of 152 protein quantification results. Log2 fold changes were calculated and proteins were considered significantly altered in expression when FDR-adjusted $p<0.1$ were obtained. Volcano plots were made from filtered data, with the biosynthetic gene clusters color-coded.

\section{Proteomics of Streptomyces sp. MBT84 expressing catechol dioxygenases} Heterologous expression of catechol dioxygenases

Constructs for the heterologous expression of catechol 1,2-dioxygenase (C12O) and catechol 2,3-dioxygenase (C23O) were constructed as follows The catA1 gene encoding $\mathrm{C} 12 \mathrm{O}$ was amplified from the genomic DNA of Pseudomonas putida KT2440 using the primer pair SF14_catA1_F and catA1_T0_R. The $x y I E$ gene encoding C23O was amplified from promoterprobe vector plJ4083 using the primer pair SF14_xylE_F and xylE_T0_R. The forward primers contain the sequence of the strong constitutive promoter SF14 40 including RBS and the reverse primers contain a t0 terminator sequence. The PCR products SF14-catA1-T0 and SF14- $x y I E-T 0$ were placed into the BamHI / Xbal site of the multi-copy vector pWHM3 to create vectors pGWS1519 and pGWS1520 respectively. These constructs were introduced into Streptomyces sp. MBT84 via protoplast transformation, whereby the empty vector was used as a control. An overview of the constructs and oligonucleotides is presented in Table S1 and Table S2. For all experiments, the growth medium was supplemented with $10 \mu \mathrm{g} / \mathrm{mL}$ thiostrepton.

\section{Analysis of the recombinant strains by quantitative proteomics}

Quantitative proteomics was used to analyse the expression of catechol dioxygenases and the expression profile of BGC1.4 in recombinant strains of Streptomyces sp. MBT84 as described previously ${ }^{41}$. Briefly, the desalted peptide solution was separated on an UltiMate 3000 RSLCnano system (Thermo Scientific) set in a trap-elute configuration, coupled to QExactive 
HF (Thermo Scientific) mass spectrometer. The LC system used a Waters nanoEase M/Z Symmetry $\mathrm{C}_{18}$ trap column $(5 \mu \mathrm{m}, 100 \AA, 180 \mu \mathrm{m} \times 20 \mathrm{~mm})$ for peptide loading/retention, and Waters nanoEase M/Z HSS T3 $\mathrm{C}_{18}$ analytical column $(1.8 \mu \mathrm{m}, 100 \AA$, $75 \mu \mathrm{m} \times 250 \mathrm{~mm})$ for peptide separation. The MS was operated in positive mode with data dependent acquisition and default charge of 2. Raw LC-MS/MS files were analysed using MaxQuant software $(v 1.6 .17 .0)^{52}$ with label free quantification (LFQ) method applied. Proteins were considered significantly altered in expression when FDR-adjusted $p<0.1$ were obtained.

\section{CRISPRi technology}

To knock down the expression of genes of the angucycline BGC, we applied CRISPRi RNA interference technology. The CRISPRi system was modified from pCRISPR-dCas9 ${ }^{53}$ by expressing Cas9 from the constitutive gapdh promoter, using vector pSET152 that integrates at the øC31 attachment site on the $S$. coelicolor chromosome ${ }^{54}$. For this, a $20 \mathrm{nt}$ spacer sequence was introduced into the sgRNA scaffold by PCR using forward primers KS $\beta$ _TF or KS $\beta$ NT2F together with reverse primer SgTermi_R_B. The PCR products were cloned into pGWS1370 55 via Ncol / BamHI restriction sites. The resulting constructs pGWS1516 (targeting template strand of KS $\beta$, control) and pGWS1517 (targeting non-template strand of KS $\beta$ ) were then introduced into Streptomyces sp. MBT84 via protoplast transformation ${ }^{45}$. An overview of the constructs and oligonucleotides is presented in Table S1 and S2.

\section{ACKNOWLEDGEMENTS}

We are grateful to Melanie Ofman for help with testing strains for adrenaline response.

\section{AUTHOR CONTRIBUTIONS}

D.A.v.B. , S.S.E., D.C., I.N., A.M.R., and V.C. collected the data and aided in data analysis. D.A.v.B., L.Z., H.P.S., and G.P.v.W. designed the experiments. G.P.v.W. supervised the research. D.A.v.B., H.P.S. and G.P.v.W. wrote the manuscript with input from all coauthors. 
612 The authors declare no competing interests.

613

614

615

616

617

618

619

620

621

622

623

624

625

626

627

628

629

630

631

632

633

634

635

636

637

638

639

640

641

642

643

644

645

646

\section{REFERENCES}

\section{References}

1. Barka, E.A. et al. Taxonomy, physiology, and natural products of Actinobacteria. Microbiol Mol Biol Rev 80, 1-43 (2016).

2. Berdy, J. Bioactive microbial metabolites. J Antibiot (Tokyo) 58, 1-26 (2005).

3. Newman, D.J. \& Cragg, G.M. Natural products as sources of new drugs over the last 25 years. J Nat Prod 70, 461-477 (2007).

4. Davies, J. \& Davies, D. Origins and evolution of antibiotic resistance. Microbiol Mol Biol Rev 74, 417-433 (2010).

5. Chevrette, M.G. et al. The antimicrobial potential of Streptomyces from insect microbiomes. Nat Commun 10, 516 (2019).

6. Culp, E.J. et al. Evolution-guided discovery of antibiotics that inhibit peptidoglycan remodelling. Nature 578, 582-587 (2020).

7. $\mathrm{Wu}, \mathrm{C}$. et al. Lugdunomycin, an angucycline-derived molecule with unprecedented chemical architecture. Angew Chem Int Ed Eng/ 58, 2809-2814 (2019).

8. Bentley, S.D. et al. Complete genome sequence of the model actinomycete Streptomyces coelicolor A3(2). Nature 417, 141-147 (2002).

9. Omura, S. et al. Genome sequence of an industrial microorganism Streptomyces avermitilis: deducing the ability of producing secondary metabolites. Proc Natl Acad Sci U S A 98, 12215-12220 (2001).

10. van der Heul, H.U., Bilyk, B.L., McDowall, K.J., Seipke, R.F. \& van Wezel, G.P. Regulation of antibiotic production in Actinobacteria: new perspectives from the postgenomic era. Nat Prod Rep 35, 575-604 (2018).

11. van Bergeijk, D.A., Terlouw, B.R., Medema, M.H. \& van Wezel, G.P. Ecology and genomics of Actinobacteria: new concepts for natural product discovery. Nat Rev Microbiol (2020).

12. van der Meij, A., Worsley, S.F., Hutchings, M.I. \& van Wezel, G.P. Chemical ecology of antibiotic production by actinomycetes. FEMS Microbiol Rev 41, 392-416 (2017).

13. Wright, E.S. \& Vetsigian, K.H. Inhibitory interactions promote frequent bistability among competing bacteria. Nat Commun 7, 11274 (2016).

14. Abrudan, M.I. et al. Socially mediated induction and suppression of antibiosis during bacterial coexistence. Proc Natl Acad Sci U S A 112, 11054-9 (2015). 
15. Sadeghi, A. et al. Diversity of the ectoines biosynthesis genes in the salt tolerant Streptomyces and evidence for inductive effect of ectoines on their accumulation. Microbiol Res 169, 699-708 (2014).

16. Kramer, J., Ozkaya, O. \& Kummerli, R. Bacterial siderophores in community and host interactions. Nat Rev Microbiol 18, 152-163 (2020).

17. Hoskisson, P.A. \& Fernandez-Martinez, L.T. Regulation of specialised metabolites in Actinobacteria - expanding the paradigms. Environ Microbiol Rep 10, 231-238 (2018).

18. Bibb, M.J. Regulation of secondary metabolism in streptomycetes. Curr Opin Microbio/ 8, 208-215 (2005).

19. Traxler, M.F., Watrous, J.D., Alexandrov, T., Dorrestein, P.C. \& Kolter, R. Interspecies interactions stimulate diversification of the Streptomyces coelicolor secreted metabolome. mBio 4 (2013).

20. Rigali, S. et al. Feast or famine: the global regulator DasR links nutrient stress to antibiotic production by Streptomyces. EMBO Rep 9, 670-5 (2008).

21. van der Meij, A. et al. Inter- and intracellular colonization of Arabidopsis roots by endophytic actinobacteria and the impact of plant hormones on their antimicrobial activity. Antonie Van Leeuwenhoek 111, 679-690 (2018).

22. Lyte, M. \& Ernst, S. Catecholamine induced growth of gram negative bacteria. Life Sci 50, 203-12 (1992).

23. Belay, T., Aviles, H., Vance, M., Fountain, K. \& Sonnenfeld, G. Catecholamines and in vitro growth of pathogenic bacteria: enhancement of growth varies greatly among bacterial species. Life Sci 73, 1527-1535 (2003).

24. Sandrini, S., Alghofaili, F., Freestone, P. \& Yesilkaya, H. Host stress hormone norepinephrine stimulates pneumococcal growth, biofilm formation and virulence gene expression. BMC Microbiol 14, 180 (2014).

25. Peterson, G., Kumar, A., Gart, E. \& Narayanan, S. Catecholamines increase conjugative gene transfer between enteric bacteria. Microb Pathog 51, 1-8 (2011).

26. Zhu, H. et al. Eliciting antibiotics active against the ESKAPE pathogens in a collection of actinomycetes isolated from mountain soils. Microbiology (Reading) 160, 17141725 (2014).

27. Bernatoniene, J. \& Kopustinskiene, D.M. The role of catechins in cellular responses to oxidative stress. Molecules 23 (2018).

28. Xia, J., Psychogios, N., Young, N. \& Wishart, D.S. MetaboAnalyst: a web server for metabolomic data analysis and interpretation. Nucleic Acids Res 37, W652-60 (2009).

29. Mladic, M. et al. Detection and identification of antibacterial proteins in snake venoms using at-line nanofractionation coupled to LC-MS. Toxicon 155, 66-74 (2018). 
30. Wang, M. et al. Sharing and community curation of mass spectrometry data with Global Natural Products Social Molecular Networking. Nat Biotechnol 34, 828-837 (2016).

31. Frank, A.M. et al. Clustering millions of tandem mass spectra. J Proteome Res 7, 11322 (2008).

32. Peng, A. et al. Angucycline glycosides from an intertidal sediments strain Streptomyces sp. and their cytotoxic activity against hepatoma carcinoma cells. Mar Drugs 16, 470 (2018).

33. Blin, K. et al. antiSMASH 6.0: improving cluster detection and comparison capabilities. Nucleic Acids Res 49, W29-W35 (2021).

34. Gubbens, J. et al. Natural product proteomining, a quantitative proteomics platform, allows rapid discovery of biosynthetic gene clusters for different classes of natural products. Chem Biol 21, 707-18 (2014).

35. Kharel, M.K. et al. Angucyclines: Biosynthesis, mode-of-action, new natural products, and synthesis. Nat Prod Rep 29, 264-325 (2012).

36. Qi, L.S. et al. Repurposing CRISPR as an RNA-guided platform for sequence-specific control of gene expression. Cell 152, 1173-83 (2013).

37. Tong, Y., Charusanti, P., Zhang, L., Weber, T. \& Lee, S.Y. CRISPR-Cas9 Based Engineering of Actinomycetal Genomes. ACS Synth Biol 4, 1020-9 (2015).

38. Ladino-Orjuela, G., Gomes, E., da Silva, R., Salt, C. \& Parsons, J.R. Metabolic pathways for degradation of aromatic hydrocarbons by bacteria. Rev Environ Contam Toxicol 237, 105-21 (2016).

39. Ingram, C., Brawner, M., Youngman, P. \& Westpheling, J. xylE functions as an efficient reporter gene in Streptomyces spp.: use for the study of galP1, a catabolitecontrolled promoter. J Bacteriol 171, 6617-24 (1989).

40. Labes, G., Bibb, M. \& Wohlleben, W. Isolation and characterization of a strong promoter element from the Streptomyces ghanaensis phage I19 using the gentamicin resistance gene (aacC1) of Tn 1696 as reporter. Microbiology (Reading) 143 ( Pt 5), 1503-1512 (1997).

41. Zhang, Z. et al. Antibiotic production in Streptomyces is organized by a division of labor through terminal genomic differentiation. Sci Adv 6, eaay5781 (2020).

42. Wu, C. et al. Expanding the chemical space for natural products by AspergillusStreptomyces co-cultivation and biotransformation. Sci Rep 5, 10868 (2015).

43. Xu, F., Nazari, B., Moon, K., Bushin, L.B. \& Seyedsayamdost, M.R. Discovery of a cryptic antifungal compound from Streptomyces albus J1074 using high-throughput elicitor screens. J Am Chem Soc 139, 9203-9212 (2017). 
44. Stubbendieck, R.M. et al. Competition among nasal bacteria suggests a role for siderophore-mediated interactions in shaping the human nasal microbiota. Appl Environ Microbio/ 85 (2019).

45. Kieser, T., Bibb, M.J., Buttner, M.J., Chater, K.F. \& Hopwood, D.A. Practical Steptomyces Genetics (John Innes Foundation, Norwich, UK, 2000).

46. Liu, M. \& Douthwaite, S. Activity of the ketolide telithromycin is refractory to Erm monomethylation of bacterial rRNA. Antimicrob Agents Chemother 46, 1629-33 (2002).

47. Schwyn, B. \& Neilands, J.B. Universal chemical assay for the detection and determination of siderophores. Anal Biochem 160, 47-56 (1987).

48. Pluskal, T., Castillo, S., Villar-Briones, A. \& Oresic, M. MZmine 2: modular framework for processing, visualizing, and analyzing mass spectrometry-based molecular profile data. BMC Bioinformatics 11, 395 (2010).

49. Myers, O.D., Sumner, S.J., Li, S., Barnes, S. \& Du, X. One step forward for reducing false positive and false negative compound identifications from mass spectrometry metabolomics data: new algorithms for constructing extracted ion chromatograms and detecting chromatographic peaks. Anal Chem 89, 8696-8703 (2017).

50. Kolmogorov, M., Yuan, J., Lin, Y. \& Pevzner, P.A. Assembly of long, error-prone reads using repeat graphs. Nat Biotechno/ 37, 540-546 (2019).

51. Distler, U. et al. Drift time-specific collision energies enable deep-coverage dataindependent acquisition proteomics. Nat Methods 11, 167-70 (2014).

52. Cox, J. \& Mann, M. MaxQuant enables high peptide identification rates, individualized p.p.b.-range mass accuracies and proteome-wide protein quantification. Nat Biotechnol 26, 1367-72 (2008).

53. Tong, Y., Charusanti, P., Zhang, L., Weber, T. \& Lee, S.Y. CRISPR-Cas9 Based Engineering of Actinomycetal Genomes. ACS Synth Biol 4, 1020-1029 (2015).

54. Ultee, E. et al. Teichoic acids anchor distinct cell wall lamellae in an apically growing bacterium. Commun Biol 3, 314 (2020).

55. Zhang, L. et al. An Alternative and Conserved Cell Wall Enzyme That Can Substitute for the Lipid II Synthase MurG. mBio 12 (2021). 


\section{FIGURE LEGENDS}

Figure 1. The catechol-moiety is key to the eliciting effect of epinephrine on Streptomyces sp. MBT42. A) Streptomyces sp. MBT42 was grown on NA supplemented with and without $100 \mu \mathrm{M}$ epinephrine. After 4 days of growth, plates were overlaid with $B$. subtilis to test for antimicrobial activity. Note the increased semi-transparent halo surrounding MBT42 grown in presence of epinephrine. B) An overview of the structurally-related compounds tested. C) Compounds with a catechol-moiety (blue columns) increase the bioactivity of Streptomyces sp. MBT42 grown on NA against $B$. subtilis, indicating that catechol is key to the eliciting effect of epinephrine.

Figure 2. Catechol elicits siderophore production by Streptomyces sp. MBT42. MBT42 was grown on NA with and without $100 \mu \mathrm{M}$ catechol, supplemented with various metal salts (5 $\mu \mathrm{M})$. After 4 days of growth, plates were overlaid with $B$. subtilis (left) to test for antimicrobial activity or with CAS agar to detect the extracellular production of iron-chelating molecules (orange halos; right). Note that catechol inhibits growth of $B$. subtilis and induces siderophore production, and that these zones are highly comparable. When iron was added to the medium, siderophore production was almost completely inhibited and the semi-transparent halo was no longer visible.

Figure 3. Catechol elicits the bioactivity and metabolite production of Streptomyces sp. MBT84. A) The antibacterial activity against B. subtilis 168 and yellow pigment production of Streptomyces sp. MBT84 are increased in the presence of catechol. B) None of the structurally-related compounds, including epinephrine, increased the antibiotic production by Streptomyces sp. MBT84. C) LC-MS chromatogram overlay of the crude extract of MBT84 grown with and without catechol. Multiple peaks were increased in intensity in the presence of catechol. D) Volcano plot highlighting the increased metabolite production by Streptomyces sp. MBT84 in response to catechol. The $\mathrm{x}$ and $\mathrm{y}$ axes represent the $\log _{2}$ 
780

781

782

fold changes and the corresponding $-\log _{10}$ FDR-adjusted $p$-value of all the mass features, respectively. Red circles represent the mass features in catechol-grown cultures with a significant intensity difference of more than 2-fold compared to control cultures (FDR-adjusted $p \leq 0.1$ ). Circles situated in the top left and top right quadrants represent the mass features which are most induced or repressed, respectively, by catechol with high statistical significance. The $\mathrm{m} / \mathrm{z}$ values 469.149 and 579.186 are shown in purple and green, respectively.

Figure 4. Nanofractionation reveals induction of several bioactive metabolites in Streptomyces sp. MBT84 by catechol. A) Bioactivity chromatogram overlay of the nanofractionated control and catechol-grown extracts. The negative peaks represent the bioactive compounds. The results of the resazurin reduction assay have been plotted against their corresponding LC-MS chromatograms after adjusting for the delay. B) Total ion current LC-MS chromatogram overlay of control and catechol-grown extracts. C-J) Extracted ion chromatogram overlay of $m / z$ values 487.16 (C), 469.15 (D), $740.20(E), 597.24(F), 579.19(G), 597.20(H), 415.21(\mathrm{I})$, and $451.14(\mathrm{~J})$ in both control (dashed line) and catechol-grown (solid line) extracts, which could be aligned to the negative maxima of the bioactivity chromatogram.

\section{Figure 5. Molecular network of the ions detected in the crude extracts of} Streptomyces sp. MBT84 revealing a large spectral family of angucyline compounds elicited by catechol. A pie chart was mapped to the nodes which represents the abundance of each $\mathrm{m} / \mathrm{z}$ value in the control (blue) and catechol-grown crude extracts (yellow). Nodes highlighted in red represent dereplicated metabolites, while those highlighted in green represent the compounds isolated and identified in 
805 this study. Streptomyces sp. MBT84 was grown for five days on MM agar plates with 806 or without $100 \mu \mathrm{M}$ catechol.

807

Figure 6. Identification of the BGC responsible for the production of angucycline glycosides in Streptomyces sp. MBT84 using antiSMASH and MS-based quantitative proteomics. A) KnownClusterBlast output from antiSMASH which shows

811 similar clusters from the MIBiG database. Genes marked with the same colour are 812 interrelated; white genes have no relationship. B) Volcano plot of MS-based 813 quantitative proteomics for cultures grown with and without catechol. Proteins with an 814 FDR-adjusted $p$-value $\geq 0.1$ are grayed out. Proteins with a positive $\log _{2}$ fold change 815 are higher expressed in catechol-grown cultures. C) BGC1.4 coding for the 816 biosynthesis of angucycline glycosides. Annotations are based on BLAST homology 817 searches. Significantly differentially expressed genes are depicted in bold (FDR818 adjusted $p<0.1)$. 


\section{Figures}

A

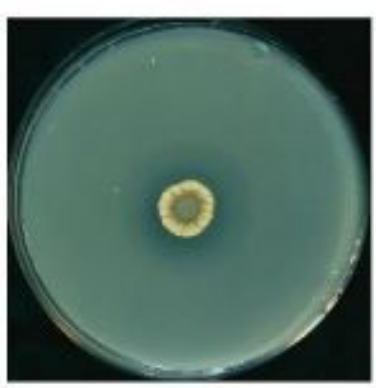

Control

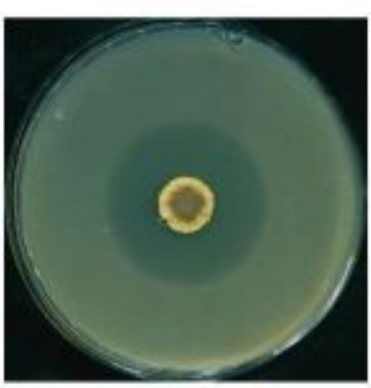

100 HM Epinephrine
B

Catechol<smiles>Oc1cc(O)c2c(c1)OC(c1ccc(O)c(O)c1)C(O)C2</smiles>

Catechin<smiles>NCCc1ccc(O)c(O)c1</smiles>

Dopamine<smiles>NC(Cc1ccc(O)c(O)c1)C(=O)O</smiles>

levodopa<smiles>CNCC(O)c1ccc(O)c(O)c1</smiles>

Epinephrine<smiles>NCC(O)c1ccc(O)c(O)c1</smiles>

Norepinephrine<smiles>Oc1ccc(O)cc1</smiles>

Hydroquinone<smiles>Oc1ccccc1</smiles>

Phenol<smiles>CNCC(O)c1cccc(O)c1</smiles>

Phenylephrine<smiles>Oc1cccc(O)c1</smiles>

Resorcinol<smiles>NCCc1ccc(O)cc1</smiles>

Tyramine

C

Bioactivity of Streptomyces sp. MBT42 against B. subtilis

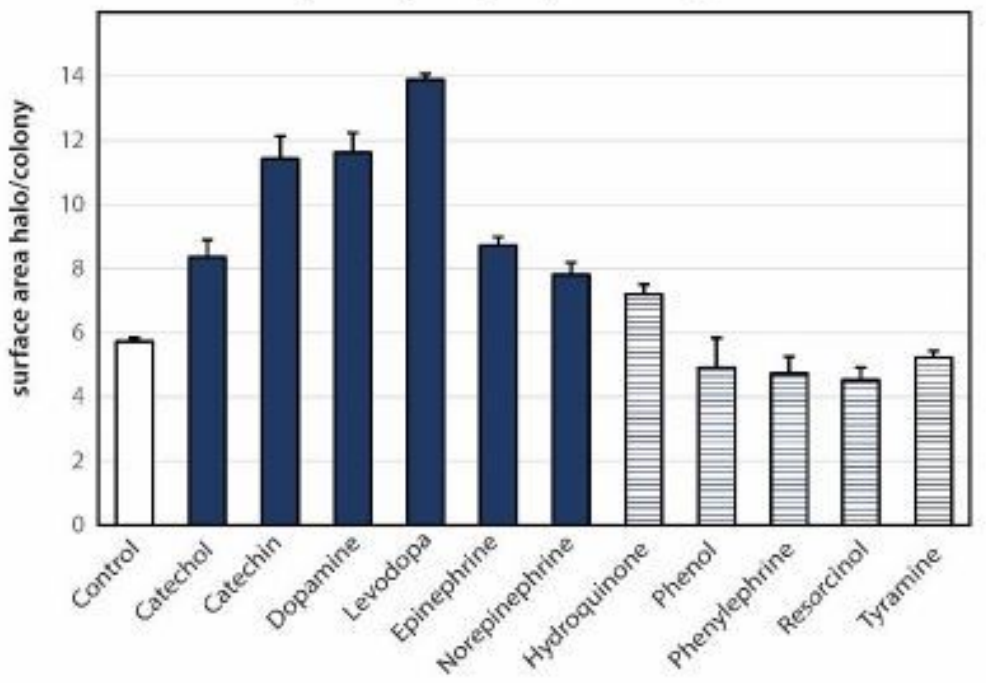

Figure 1

The catechol-moiety is key to the eliciting effect of epinephrine on Streptomyces sp. MBT42. A) Streptomyces sp. MBT42 was grown on NA supplemented with and without $100 \mu \mathrm{M}$ epinephrine. After 4 days of growth, plates were overlaid with B. subtilis to test for antimicrobial activity. Note the increased semi-transparent halo surrounding MBT42 grown in presence of epinephrine. B) An overview of the structurally-related compounds tested. C) Compounds with a catechol-moiety (blue columns) increase the 
bioactivity of Streptomyces sp. MBT42 grown on NA against B. subtilis, indicating that catechol is key to the eliciting effect of epinephrine.

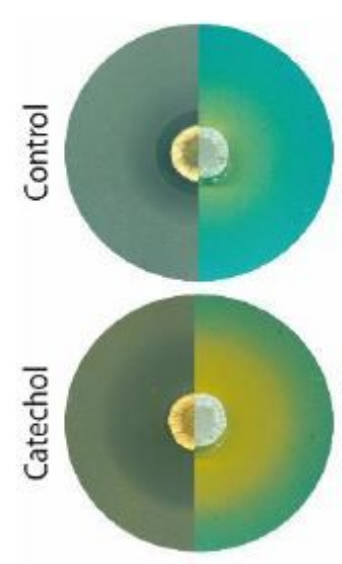

$-$

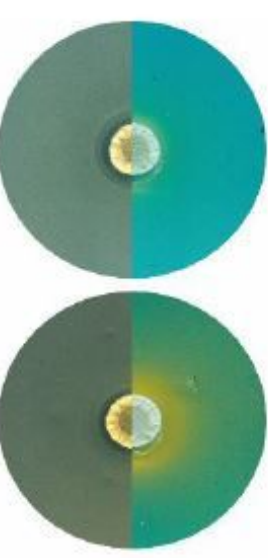

$\mathrm{FeCl}_{3}$

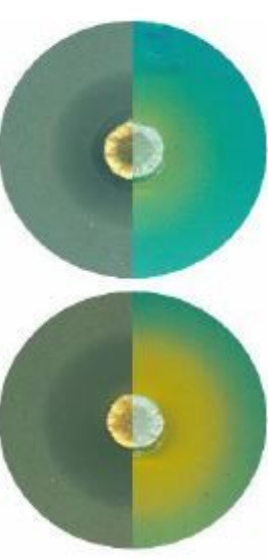

$\mathrm{ZnCl}_{2}$

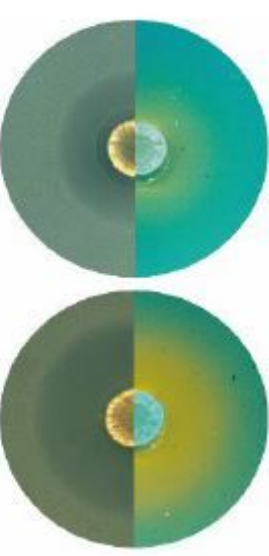

$\mathrm{MnCl}_{2}$

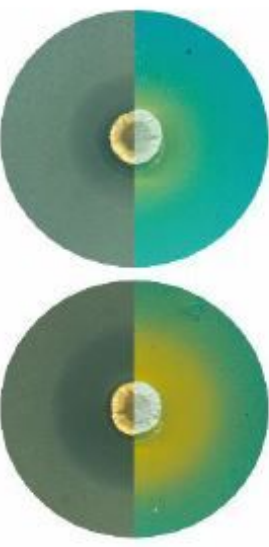

$\mathrm{CoCl}_{2}$

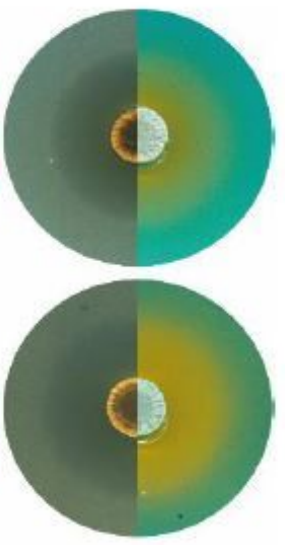

$\mathrm{CuSO}_{4}$

\section{Figure 2}

Catechol elicits siderophore production by Streptomyces sp. MBT42. MBT42 was grown on NA with and without $100 \mu \mathrm{M}$ catechol, supplemented with various metal salts $(5 \mu \mathrm{M})$. After 4 days of growth, plates were overlaid with B. subtilis (left) to test for antimicrobial activity or with CAS agar to detect the extracellular production of iron-chelating molecules (orange halos; right). Note that catechol inhibits growth of B. subtilis and induces siderophore production, and that these zones are highly comparable. When iron was added to the medium, siderophore production was almost completely inhibited and the semi-transparent halo was no longer visible. 

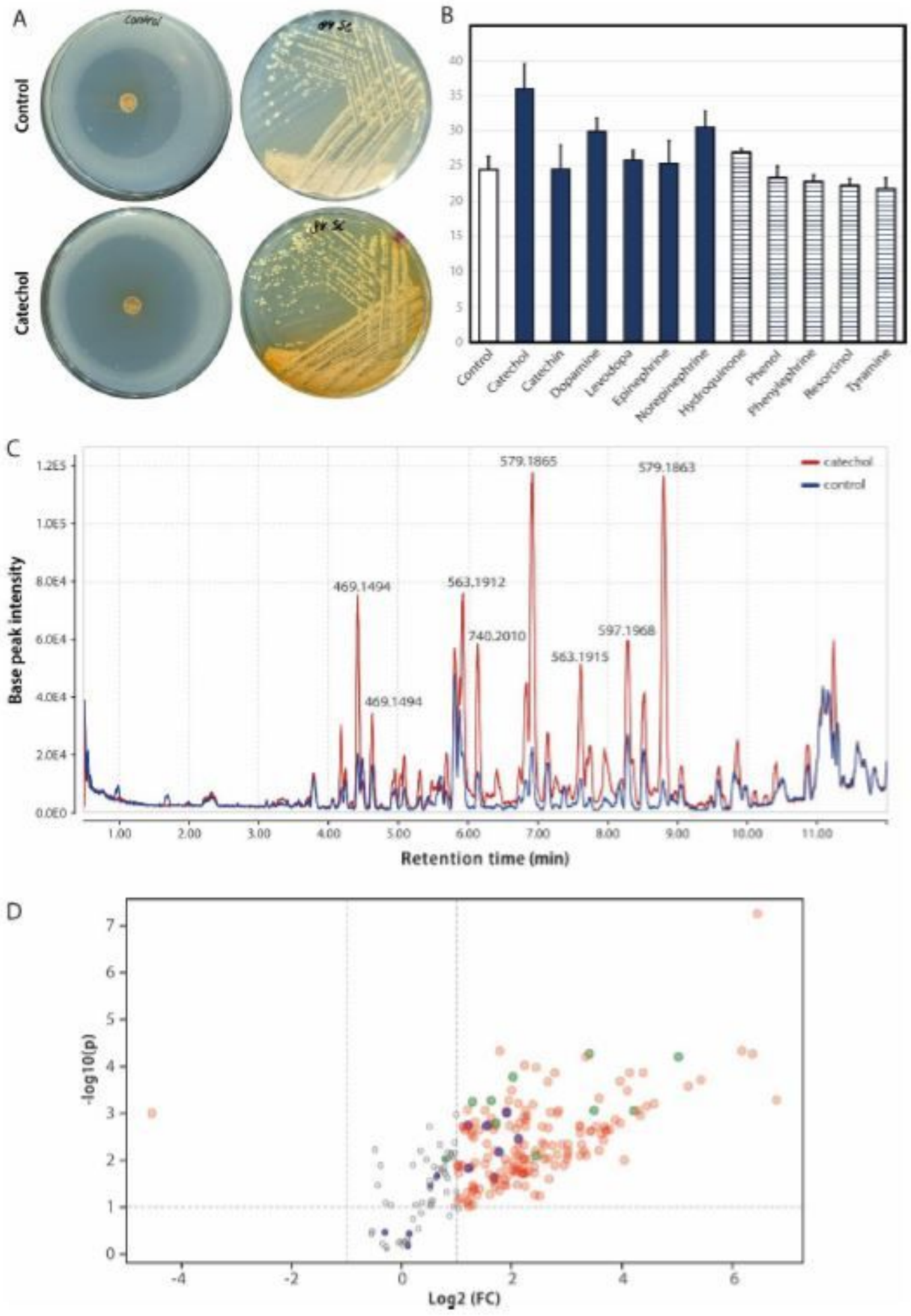

\section{Figure 3}

Catechol elicits the bioactivity and metabolite production of Streptomyces sp. MBT84. A) The antibacterial activity against B. subtilis 168 and yellow pigment production of Streptomyces sp. MBT84 are increased in the presence of catechol. B) None of the structurally-related compounds, including epinephrine, increased the antibiotic production by Streptomyces sp. MBT84. C) LC-MS chromatogram overlay of the crude extract of MBT84 grown with and without catechol. Multiple peaks were increased in 
intensity in the presence of catechol. D) Volcano plot highlighting the increased metabolite production by Streptomyces sp. MBT84 in response to catechol. The $x$ and $y$ axes represent the log2 29 fold changes and the corresponding - log10 FDR-adjusted p-value of all the mass features, respectively. Red circles represent the mass features in catechol-grown cultures with a significant intensity difference of more than 2-fold compared to control cultures (FDR-adjusted $p \leq 0.1$ ). Circles situated in the top left and top right quadrants represent the mass features which are most induced or repressed, respectively, by catechol with high statistical significance. The $\mathrm{m} / \mathrm{z}$ values 469.149 and 579.186 are shown in purple and green, respectively.

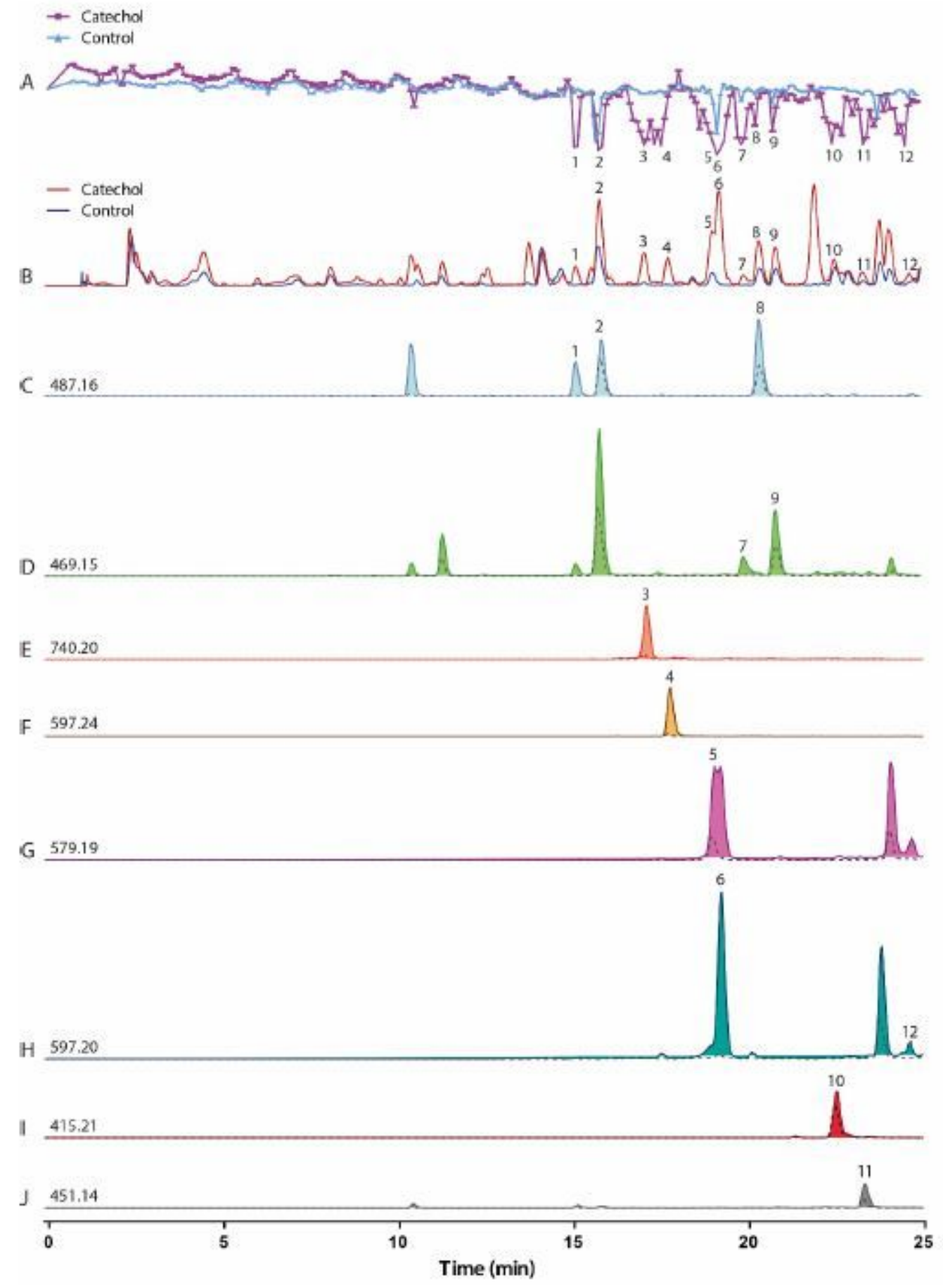

Figure 4 
Nanofractionation reveals induction of several bioactive metabolites in Streptomyces sp. MBT84 by catechol. A) Bioactivity chromatogram overlay of the nanofractionated control and catechol-grown extracts. The negative peaks represent the bioactive compounds. The results of the resazurin reduction assay have been plotted against their corresponding LC-MS chromatograms after adjusting for the delay. B) Total ion current LC-MS chromatogram overlay of control and catechol-grown extracts. C-J) Extracted ion chromatogram overlay of $\mathrm{m} / \mathrm{z}$ values 487.16 (C), 469.15 (D), 740.20 (E), 597.24 (F), 579.19 (G), $597.20(\mathrm{H}), 415.21(\mathrm{I})$, and $451.14(\mathrm{~J})$ in both control (dashed line) and catechol-grown (solid line) extracts, which could be aligned to the negative maxima of the bioactivity chromatogram.

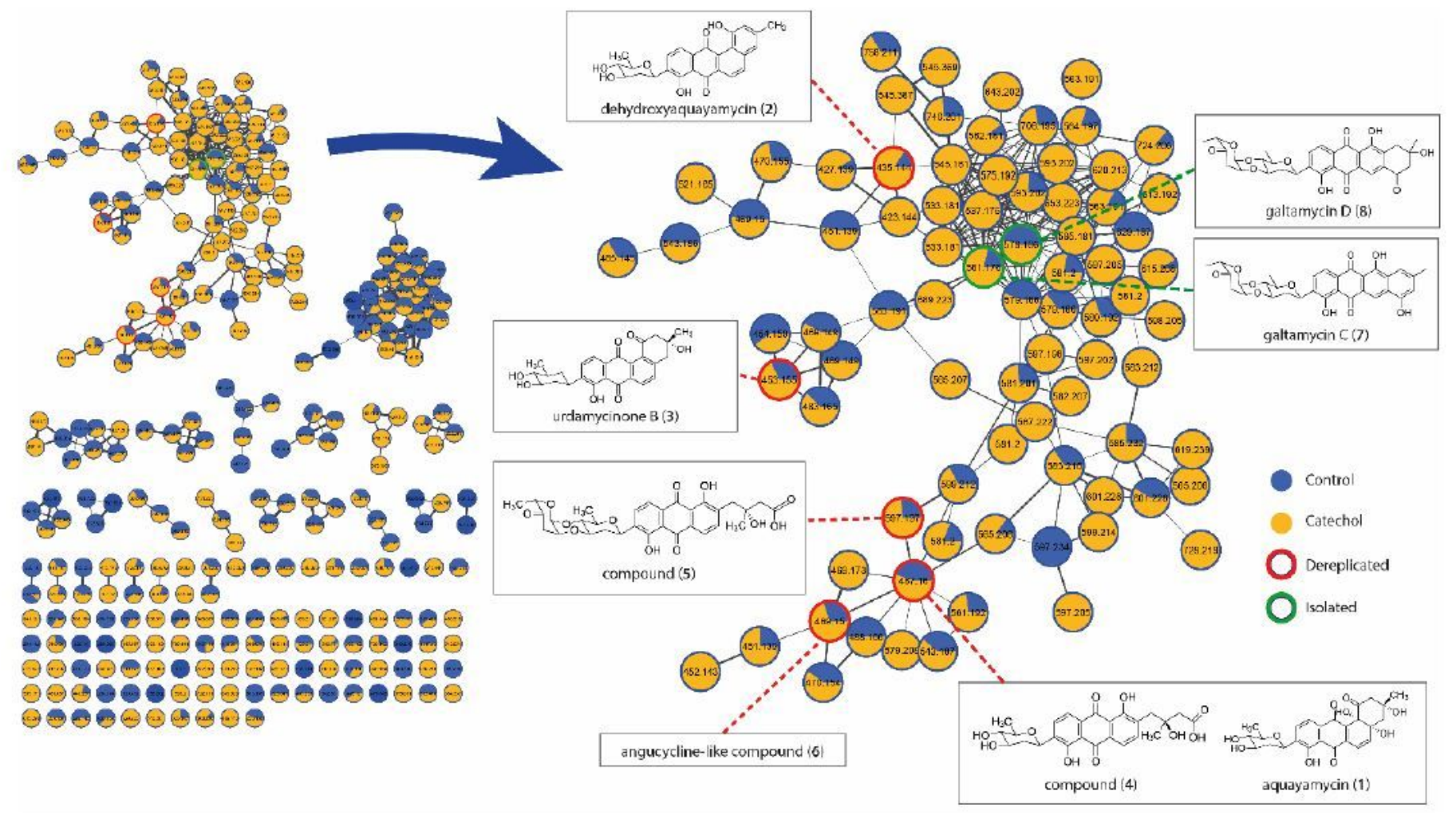

\section{Figure 5}

Molecular network of the ions detected in the crude extracts of Streptomyces sp. MBT84 revealing a large spectral family of angucyline compounds elicited by catechol . A pie chart was mapped to the nodes which represents the abundance of each $\mathrm{m} / \mathrm{z}$ value in the control (blue) and catechol-grown crude extracts (yellow). Nodes highlighted in red represent dereplicated metabolites, while those highlighted in green represent the compounds isolated and identified in this study. Streptomyces sp. MBT84 was grown for five days on MM agar plates with or without $100 \mu \mathrm{M}$ catechol. 
MBT84 BGC1.4

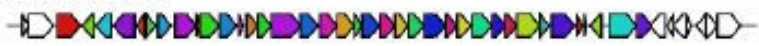

BGC0001769: saquayamycin A (87\% of genes show similarity)

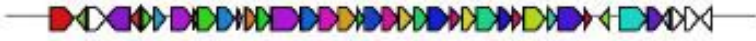

BGC0001384: saprolmycin E (91\% of genes show similarity)

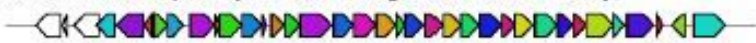

BGC0000229: grincamycin ( $88 \%$ of genes show similarity)

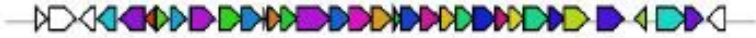

BGC0000268: Sch-47554/ Sch-47555 (52\% of genes show similarity)

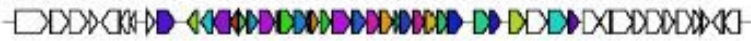

BGC0000267: saquayamycin Z (73\% of genes show similarity)

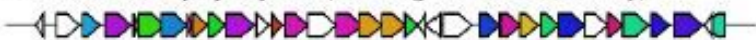

BGC0000262: prejadomycin/rabelomycin/ UWM6 ( $52 \%$ of genes show similarity)

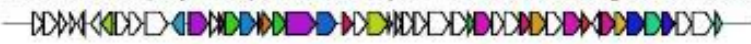

BGC0000239: landomycin A (71\% of genes show similarity)

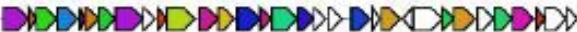

BGCOO01438: warkmycin CS1 / CS2 (58\% of genes show similarity)

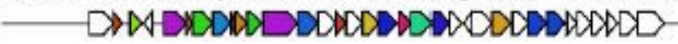

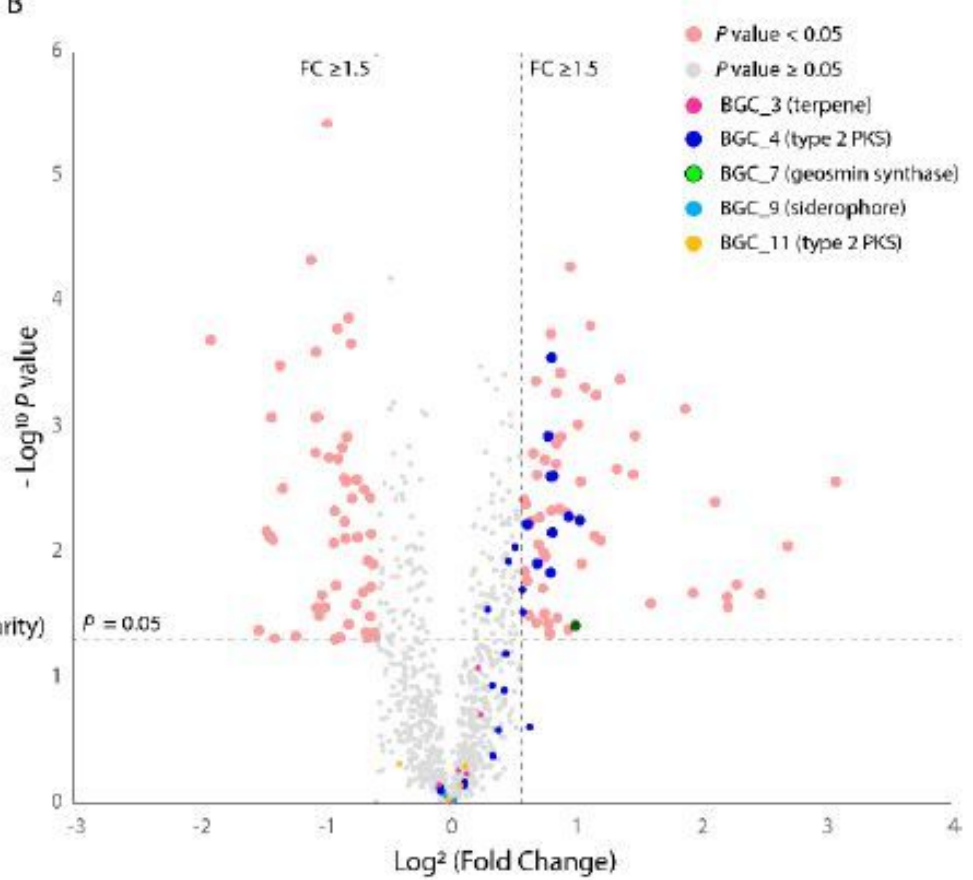

$\log ^{2}$ (Fold Change)

$D$ core biosynthetic
genes

\begin{tabular}{|c|c|c|c|c|c|c|c|}
\hline Gene & Function & $\log ^{2}(\mathrm{FC})$ & $q$-value & Gene & Function & $\log ^{2}(F C)$ & $q$-value \\
\hline 1 & Putative multidrug-efflux transporter & n.d. & n.d. & 20 & L-noviosyl transferase & 1.0 & 0.0598 \\
\hline 2 & Unknown & n.d. & n.d. & 21 & Glucose-1-phosphate thymidylyltransferase & 0.3 & 0.6248 \\
\hline 3 & $\mathrm{NADH}$ oxidase & 0.3 & 0.1366 & 22 & dTDP-glucose 4,6-dehydratase & 0.4 & 0.2099 \\
\hline 4 & Multidrug resistance protein 3 & 0.8 & 0.0351 & 23 & dTOP-6-deoxy-L-talose 4-dehydrogenase (NAC $(P][+])$ & 0.6 & 0.0610 \\
\hline 5 & Unknown & 0.7 & 0.0881 & 24 & GDP-perosamine synthase & 0.8 & 0,0263 \\
\hline 6 & Tetracycline repressor protein class E & 0.6 & 0.4529 & 25 & dTDP-glucose 4,6-dehydratase & 0.6 & 0.1119 \\
\hline 7 & Unknown & n.d. & n.d. & 26 & Unknown & 0.9 & 0.0582 \\
\hline 8 & Anhydrotetracycline monooxygenase & 0.4 & 0.4658 & 27 & Unknown & 0.8 & 0.0943 \\
\hline 9 & Tetracenomycin F2 cyclase & n.d. & n.d. & 28 & Glucose--fructose oxidoreductase & n.d. & n.d. \\
\hline 10 & Actinorhodin polyketide putative beta-ketoacyl synthase 1 & 0.3 & 0.2904 & 29 & $4^{\prime}$-phosphopantetheinyl transferase psf-1 & n.d. & n.d. \\
\hline 11 & Actinorhodin polyketide putative beta-ketoacyl synthase 2 & 0.4 & 0.3079 & 30 & putative propionyl-CoA carboxylase beta chain 5 & n.d. & n.d. \\
\hline 12 & Oxytetracycline polyketide synthase acyl carrier protein & -0.1 & 0.8929 & 31 & Unknown & n.d. & n.d. \\
\hline 13 & Putative ketoacyl reductase & 0.6 & 0.1403 & 32 & Aclacinomycin-N/aclacinomycin-A oxidase & 0.8 & 0.0454 \\
\hline 14 & Putative polyketide cyclase & 0.5 & 0.0870 & 33 & Unknown & n.d. & n.d. \\
\hline 15 & Anycortetracycline monooxygenase & 0.5 & 0.0766 & 34 & Unknown & n.d. & n.d. \\
\hline 16 & Putative MFS-type transporter EfpA & n.d. & n.d. & 35 & Transcriptional regulatory protein YycF & n.d. & n.d. \\
\hline 17 & Aclacinomycin-T 2-deoxy-L-fucose transferase & 0.8 & 0.0454 & 36 & 2-oxoglutarate oxidoreductase subunit KorA & 0.1 & 0.8118 \\
\hline 18 & L-noviosyl transferase & 0.8 & 0.0678 & 37 & 2-oxoglutarate oxidereduetase subunit KarB & n.d. & n.d. \\
\hline 19 & dTDP-4-dehydrorhamnose 3,5-epimerase & n.d. & n.d. & & & & \\
\hline
\end{tabular}

\section{Figure 6}

Identification of the BGC responsible for the production of angucycline glycosides in Streptomyces sp. MBT84 using antiSMASH and MS-based quantitative proteomics. A) KnownClusterBlast output from antiSMASH which shows similar clusters from the MIBiG database. Genes marked with the same colour are interrelated; white genes have no relationship. B) Volcano plot of MS-based quantitative proteomics for cultures grown with and without catechol. Proteins with an FDR-adjusted p-value $\geq 0.1$ are grayed out. Proteins with a positive log2 fold change are higher expressed in catechol-grown cultures. C) BGC1.4 
coding for the biosynthesis of angucycline glycosides. Annotations are based on BLAST homology searches. Significantly differentially expressed genes are depicted in bold (FDR818 adjusted $p<0.1$ ).

\section{Supplementary Files}

This is a list of supplementary files associated with this preprint. Click to download.

- vanBergeijkNatChemBiolSI.pdf 